\title{
Reliability and Validity of a Smartphone-Paired Pulse Oximeter for Screening of Critical Congenital Heart Defects in Newborns
}

\author{
Maurice J. Huizing a Eduardo Villamor-Martínez ${ }^{a, c}$ Ingrid A. Chavagne ${ }^{a}$ \\ Ward Y. Vanagt ${ }^{\text {a,d }}$ Marc A. E. Spaanderman ${ }^{b, c}$ Eduardo Villamor ${ }^{a, c}$ \\ Departments of a Pediatrics and ${ }^{b}$ Obstetrics and Gynaecology, Maastricht University Medical Center (MUMC+), \\ ${ }^{\mathrm{C}}$ GROW School for Oncology and Developmental Biology, and d Cardiovascular Research Institute Maastricht CARIM, \\ Maastricht, The Netherlands
}

\section{Keywords}

Pulse oximetry $\cdot$ Screening $\cdot$ Critical congenital heart defects · Smartphone

\begin{abstract}
Background: Barriers to widespread implementation of pulse oximetry screening of critical congenital heart defects (CCHD) in newborns include increasing trends of out-of-hospital births and cost of equipment. In recent years, smartphone-compatible pulse oximeters have appeared on the market, but the validity of such devices in the setting of CCHD screening has not been evaluated. Objectives: To compare the performance in CCHD screening of a smartphone-paired pulse oximeter (Masimo iSpO $2-\mathrm{Rx}^{\mathrm{TM}}$ ) and a hospital-grade pulse oximeter (Masimo Radical-7'M). Methods: Preductal (right hand) and postductal (either foot) saturations were determined in a population of 201 term newborns by 2 independent teams, one using the Radical-7 and the other using the iSpO $\mathrm{O}_{2}-\mathrm{Rx}$. Bland-Altman analysis was applied to calculate mean bias and $95 \%$ limits of agreement between the 2 pulse oximeters. Results: For the preductal oxygen saturation, the mean bias (Radical-7 minus iSpO $\mathrm{O}_{2}-\mathrm{Rx}$ ) was -0.08 (SD 1.76) and the lower and upper limits of agreement were -3.52 and 3.36 , respectively. For the postductal
\end{abstract}

\section{KARGER}

(C) 2017 S. Karger AG, Basel

E-Mail karger@karger.com

www.karger.com/neo oxygen saturation, the mean bias was -0.11 (SD 1.68) and the lower and upper limits of agreement were -3.49 and 3.18, respectively. In addition, the $\mathrm{iSpO}_{2}-\mathrm{Rx}$ provided reliable measurements of saturations below $95 \%$ in a group of 12 infants admitted to the neonatal intensive care unit. Conclusions: Our data suggest that CCHD screening with the Masimo iSpO $\mathrm{S}_{2}-\mathrm{Rx}$ is feasible and accurate. The use of reliable smartphone-paired pulse oximeters may contribute to the extension of CCHD screening to home births and low resource settings.

(c) 2017 S. Karger AG, Basel

\section{Introduction}

There is overwhelming evidence that pulse oximetry screening increases early detection of critical congenital heart defects (CCHD) in newborns [1-5]. Consequently, many programs and groups around the world have recommended and adopted this type of screening [4, 6-10]. However, barriers to widespread implementation of pulse oximetry screening of CCHD include increasing trends of out-of-hospital births, cost of implementation (equipment and staff costs), and management of test positives (neonatal admissions and echocardiography) [11]. 
When selecting a pulse oximeter for CCHD screening, it is recommended that the monitor should be approved for use in neonates, be motion tolerant, and be able to read through low perfusion [10, 12]. Therefore, hospital-grade pulse oximeters are used in all screening programs [2, 5-9, $13,14]$. Nevertheless, the price of such devices is a limitation for the extension of the CCHD pulse oximetry screening to home births and low-resource settings. In recent years, several manufacturers have developed low-cost pulse oximeters devices that can be paired to a smartphone or a tablet [15-17]. The inherent computing power of the mobile phone or tablet and its everyday availability offer the opportunity to create a low-cost stand-alone device which may extend pulse oximetry from hospital into nonhospital settings $[16,17]$. However, to the best of our knowledge, none of these devices has been tested in the setting of CCHD screening. In this investigator-initiated study, we compared the performance in CCHD screening of a smartphone-paired pulse oximeter (Masimo iSpO $2_{2-}^{-}$ $\mathrm{Rx}^{\mathrm{TM}}$ ) and a hospital-grade pulse oximeter (Masimo Radical-7 ${ }^{\mathrm{TM}}$ ) from the same manufacturer.

\section{Methods}

Study Subjects and Protocol

The study was approved by the local ethics committee and informed consent of the parents was obtained. All healthy term $(>37$ weeks) infants born at the Maastricht University Medical Center (MUMC) were potentially eligible for the study.

The equipment and supplies used in the study were acquired at market price by the purchasing department of MUMC. Pulse oximetry screening of CCHD was introduced in MUMC in January 2016 as part of the routine care of healthy newborns. The screening is performed by trained nurses using the Masimo Radical-7 (Masimo Corp., Irvine, CA, USA; price in the Netherlands: EUR 1,800) equipped with disposable Masimo LNCS sensors. Screening is performed 12-24 h after birth and involves preductal (right-hand) and postductal (either foot) measurements. Screenings before $12 \mathrm{~h}$ or after $24 \mathrm{~h}$ are also allowed. Whenever possible, it is attempted to screen infants awake, quiet, and without pacifier, bottle, or concurrent feeding.

The infants included in the study were subjected to a second screening with a Masimo iSpO $\mathrm{O}_{2}-\mathrm{Rx}$ pulse oximeter (price in the Netherlands: EUR 300) equipped with a reusable Masimo MLNCS YI sensor and connected to a tablet (Apple iPad mini). The $\mathrm{iSpO}_{2}-\mathrm{Rx}$ is a CE-marked, dual-wave pulse oximeter equipped with signal extraction technology (SET ${ }^{\mathrm{TM}}$ ) that, according to the manufacturer, provides reliable readings even in low-perfusion states and with patient movements $[18,19]$. The companion software application (app) provides the oxygen saturation, the pulse rate, and the perfusion index $[17,19]$. The screening with the $\mathrm{iSpO}_{2}-\mathrm{Rx}$ took place either before or after the routine screening with the Radical-7 and was performed by one of the investigators (M.J.H., I.A.C., or E.V.). The Radical-7 and the $\mathrm{iSpO}_{2}-\mathrm{Rx}$ screen-

Smartphone Pulse Oximeter ing teams proceeded independently and were unaware of each other's results.

A screening result was labeled as "fail" if any oxygen saturation measure was $\leq 89 \%$. Any screening that was $\geq 95 \%$ in either extremity with $\leq 3 \%$ absolute difference in oxygen saturation between the upper and lower extremity was considered a "pass" result, and screening was ended. When saturation in both extremities was $\geq 90 \%$ but $\leq 94 \%$ or one of the saturations was $\geq 95$ but there was a $\geq 4 \%$ absolute difference in oxygen saturation between the right hand and foot, the screening result was labeled as "repeat" and repeated after $1 \mathrm{~h}$. Two consecutive "repeat" results were considered as a positive screening. For the purpose of the study, only the result of the first measurement was taken into account.

In order to evaluate the performance of the $\mathrm{iSpO}_{2}-\mathrm{Rx}$ pulse oximeter in detecting low oxygen saturations, a group of 12 infants admitted to the neonatal intensive care unit (NICU) of the MUMC and showing a stable saturation $<95 \%$ were also included in the study. These infants were continuously monitored with an IntelliVue MP70 patient monitor (Philips Medical Systems, The Netherlands) equipped with a Masimo SET ${ }^{\mathrm{TM}}$ OEM board pulse oximeter. This pulse oximeter was maintained in one foot and the $\mathrm{iSpO}_{2}-\mathrm{Rx}$ was connected to the other foot. Oxygen saturation was simultaneously measured in both feet during $10 \mathrm{~min}$ and recorded every minute.

\section{Statistical Analysis}

Results are expressed as counts and percentages, median and interquartile range (IQR, Q1-Q3), or means and standard deviation (SD). For agreement between the two pulse oximeters, a Bland-Altman analysis was applied calculating bias as the mean difference between both pulse oximeters and limits of agreement (bias $\pm 1.96 \mathrm{SD}$ ) as the range in which $95 \%$ of the differences between the two measurements are expected to lie [20,21]. Sample size was determined based on Bland's recommendation of using a sample size of 200 subjects to accurately estimate the limits of agreement between 2 devices or methods of measurement [20].

\section{Results}

Duplicated screening with the Radical-7 and the $\mathrm{iSpO}_{2}-\mathrm{Rx}$ was performed in 201 term infants $(51 \% \mathrm{fe}-$ male) born between July 12, 2016 and February 7, 2017. Screening with the Radical-7 took place at a median time of 21.2 $\mathrm{h}$ (IQR 16.4-24.6) and screening with the $\mathrm{iSpO}_{2^{-}}$ $\mathrm{Rx}$ took place at a median time of $22.2 \mathrm{~h}$ (IQR 18.0-27.0). The median difference between the measurements with the Radical-7 and the iSpO $\mathrm{O}_{2}-\mathrm{Rx}$ was $1.2 \mathrm{~h}$ (IQR 0.3-6.1).

For the preductal oxygen saturation, the mean bias (Radical-7 minus $\mathrm{iSpO}_{2}-\mathrm{Rx}$ ) was -0.08 (SD 1.76) and the lower and upper limits of agreement (bias $\pm 1.96 \mathrm{SD}$ ) were -3.52 and 3.36, respectively (Fig. 1). For the postductal oxygen saturation, the mean bias (Radical-7 minus $\mathrm{iSpO}_{2}-\mathrm{Rx}$ ) was -0.11 (SD 1.68) and the lower and upper limits of agreement (bias $\pm 1.96 \mathrm{SD}$ ) were -3.41 and 3.18 , respectively (Fig. 2).

Neonatology 2017;112:324-329

DOI: $10.1159 / 000477294$ 
Fig. 1. Bland-Altman plots for comparison of preductal (right hand) oxygen saturation (Sat) measured by the Masimo Radical-7 and the Masimo iSpO $\mathrm{S}_{2}-\mathrm{Rx}$ pulse oximeters in 201 term infants. The middle horizontal line represents the bias (mean difference) and the outer lines represent the limits of agreement (bias $\pm 1.96 \mathrm{SD}$ ). The numbers above the points indicate the number $(>1)$ of points falling on the same place on the plot.

Fig. 2. Bland-Altman plots for comparison of postductal (either foot) oxygen saturation (Sat) measured by the Masimo Radical-7 and the Masimo iSpO $\mathrm{O}_{2}-\mathrm{Rx}$ pulse oximeters in 201 term infants. The middle horizontal line represents the bias (mean difference) and the outer lines represent the limits of agreement (bias $\pm 1.96 \mathrm{SD}$ ). The numbers above the points indicate the number $(>1)$ of points falling on the same place on the plot.
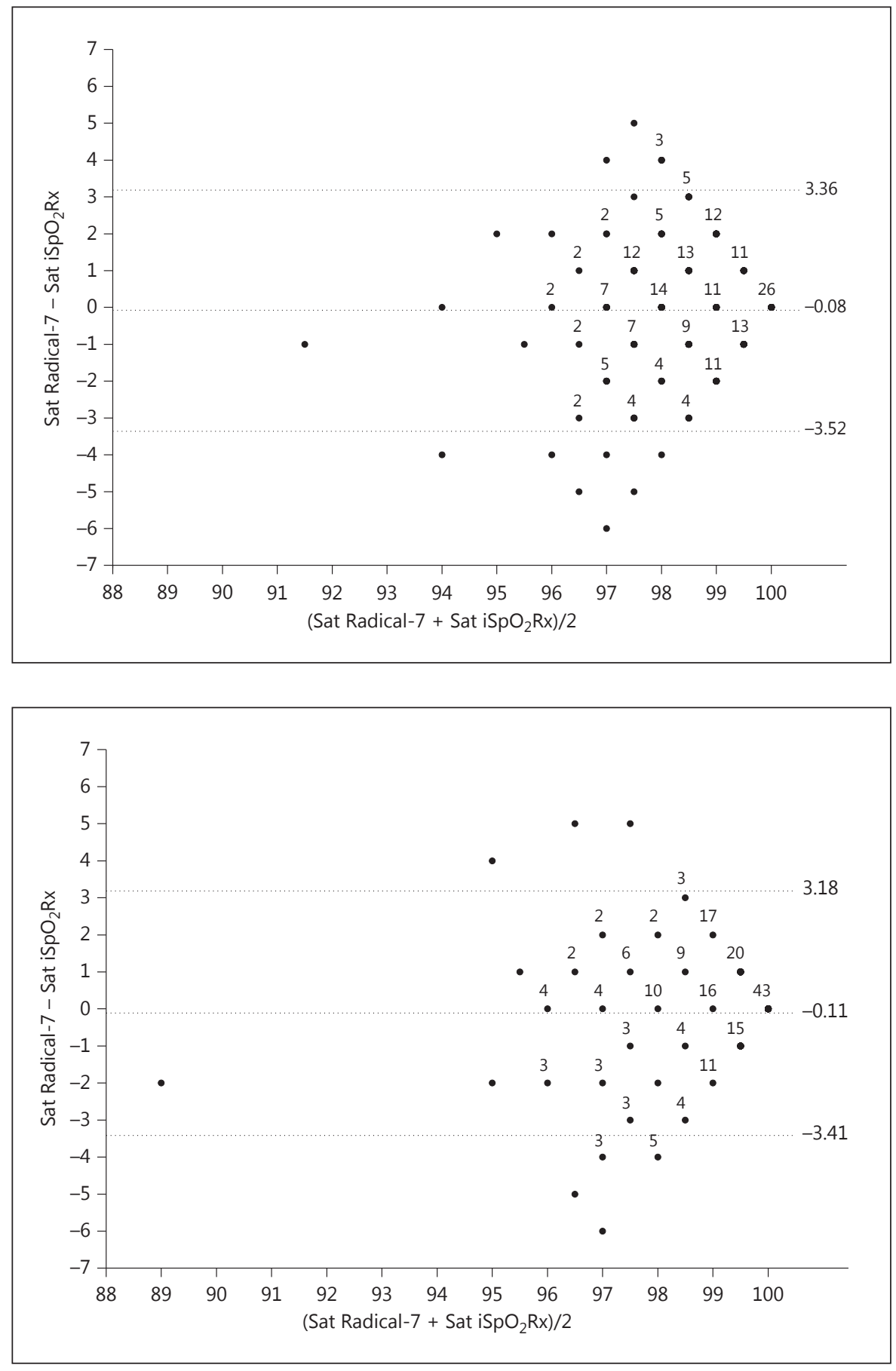

In 196 of the 201 infants (97.6\%), the screening was labeled as "pass" with both devices after the first measurement. In 5 cases the results of the first measurement were discrepant between the two pulse oximeters. Two cases were labeled as "repeat" with the Radical-7 (preductalpostductal saturations: 94-94 and 92-94) but as "pass" with the $\mathrm{iSpO}_{2}-\mathrm{Rx}$ (preductal-postductal saturations: 9496 and 96-99, respectively). Two cases were labeled as "pass" with the Radical-7 (preductal-postductal saturations: 98-97 and 98-99), but as "repeat" with the $\mathrm{iSpO}_{2}$ $\mathrm{Rx}$ (preductal-postductal saturations: $97-93$ and 100-94, respectively). Finally, in one case the infant failed the 
Fig. 3. Bland-Altman plots for comparison of postductal (either foot) oxygen saturation (Sat) measured by the Masimo SET and the Masimo iSpO $\mathrm{in}_{2}$-Rx pulse oximeters in 12 NICU infants. The middle horizontal line represents the bias (mean difference) and the outer lines represent the limits of agreement (bias $\pm 1.96 \mathrm{SD}$ ). The numbers above the points indicate the number $(>1)$ of points falling on the same place on the plot.

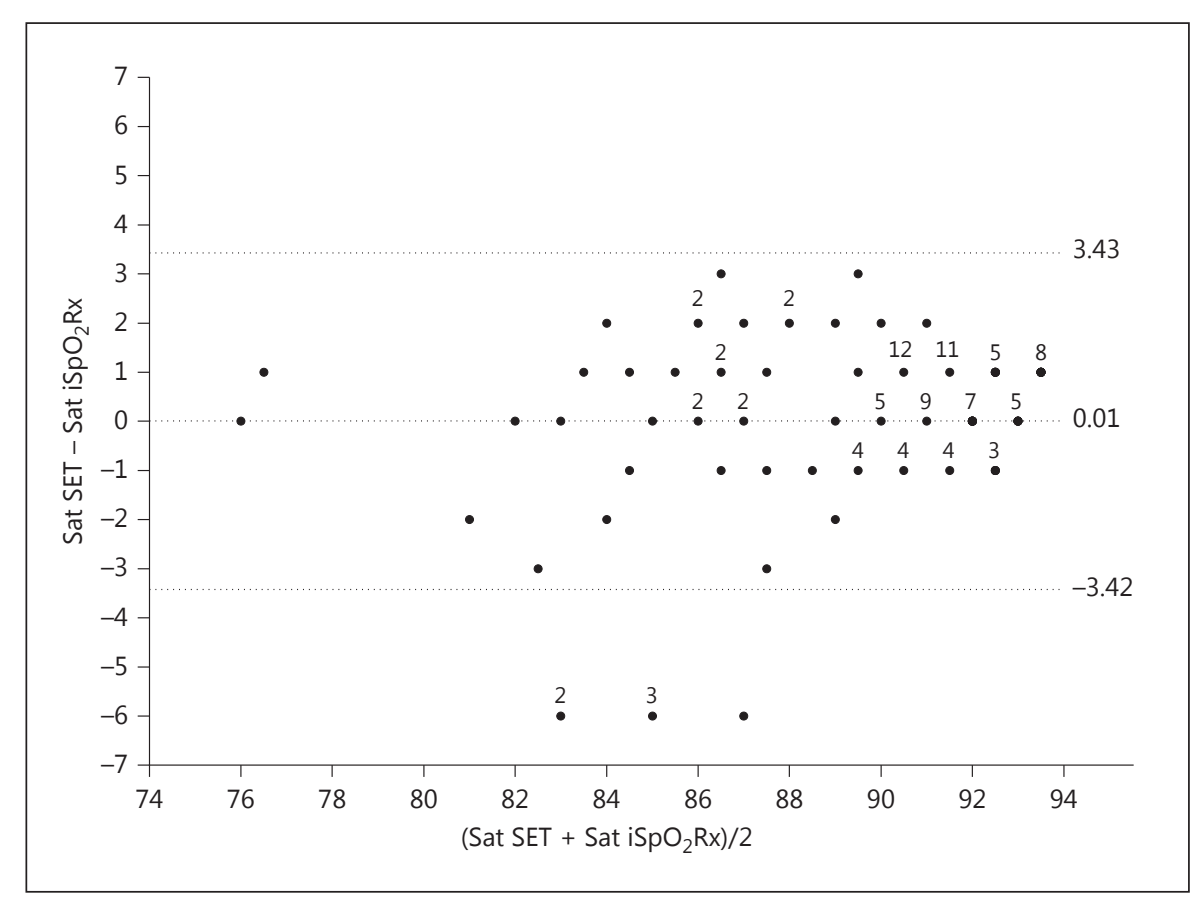

screening with the Radical-7 (preductal-postductal saturation: 91-88) but the result with the $\mathrm{iSpO}_{2}-\mathrm{Rx}$ was labeled as "repeat" (preductal-postductal saturation: 9290). This infant showed a mild respiratory distress and was immediately admitted to the neonatal unit where saturations around $90 \%$ were confirmed. No heart murmur or other abnormalities were detected by physical examination. Treatment with antibiotics was started under the diagnosis of suspected sepsis. Saturations and the clinical condition of the infant improved in the following hours and echocardiography was not considered necessary.

As mentioned in the methods section, in 12 NICU patients ( 8 with bronchopulmonary dysplasia, 2 with pulmonary hypertension, 1 with hypoplastic left ventricle and 1 with tetralogy of Fallot) under continuous monitoring with a Masimo SET pulse oximeter and showing postductal (foot) saturations $<95 \%$, we performed a simultaneous measurement of the saturation with the $\mathrm{iSpO}_{2}-\mathrm{Rx}$ in the contralateral foot. Ten measurements per infant were recorded showing a mean bias (Masimo SET minus $\mathrm{iSpO}_{2}-\mathrm{Rx}$ ) of 0.01 (SD 1.74) with lower and upper limits of agreement (bias $\pm 1.96 \mathrm{SD}$ ) of -3.42 and 3.43, respectively (Fig. 3). Visual inspection of the Bland-Altman scatterplots (Fig. 1-3) revealed no distinct patterns or funneling that would indicate important heteroscedasticity (i.e., larger variability for higher saturation values) [22].

Smartphone Pulse Oximeter

\section{Discussion}

We examined the performance of the Masimo $\mathrm{iSpO}_{2^{-}}$ $\mathrm{Rx}$, a smartphone-paired pulse oximeter, in the setting of CCHD screening in 201 newborn infants. The iSpO $\mathrm{P}_{2}-\mathrm{Rx}$ demonstrated a high degree of agreement with the Masimo Radical-7, a hospital-grade pulse oximeter. Moreover, the $\mathrm{iSpO}_{2}-\mathrm{Rx}$ provided reliable measurements of saturations below $95 \%$ in a group of NICU patients. We did not observe any systematic bias and the differences between the two pulse oximeters can be attributed to the inherent bias that all saturation monitors have when they function according to their specifications [18]. Unfortunately, we can only speculate on this point because we did not perform repeated measurements with any of the two devices in order to estimate the inherent bias in our setting. Nevertheless, it should be noted that current industry data report neonatal-based pulse oximetry algorithms to have a precision (SD of the bias) that can be as low as $2 \%$ on some monitors but as high as $5 \%$ on others [18]. Interestingly, the $\mathrm{SD}$ of the bias that we observed between the Radical-7 and the iSpO $\mathrm{O}_{2}-\mathrm{Rx}$ was less than $2 \%$. Therefore, altogether, our data suggest that CCHD screening with the $\mathrm{SpO}_{2}-\mathrm{Rx}$ is feasible and accurate.

In the Netherlands, pulse oximetry screening of CCHD has not been included in the universal screening program, but an implementation performing trial aiming to

Neonatology 2017;112:324-329 DOI: $10.1159 / 000477294$ 
screen 20,000 infants in the Amsterdam-Haarlem-Leiden region is currently underway [13]. As analyzed by Narayen et al. $[13,14]$, the perinatal health care system in the Netherlands is unique due to its high incidence of home births and early discharge after uncomplicated deliveries. In total, $33 \%$ of all low-risk deliveries are supervised by a community midwife, of which $55 \%$ occur at home and $45 \%$ at a birthing facility or policlinic $[13,14]$. Therefore, it has been estimated that implementation of a universal pulse oximetry screening of CCHD in the Netherlands would require the provision of pulse oximeters and adequate training to all 1,800 community midwives [13]. A reliable smartphone-paired pulse oximeter would offer a reduction of the cost and, simultaneously, an improvement in the quality of the screening. The latter can be achieved through the development of smartphone apps that guide the user in performing and interpreting the pulse oximetry screening.

Earlier studies have shown the feasibility of homebirth screening of CCHD with hospital-grade portable pulse oximeters $[13,14,23]$. Besides the Netherlands, rates of planned out-of-hospital birth (i.e., births intended to occur at home or at a freestanding birth center) have increased in high- and middle-income countries [24-26]. In addition, there is an increasing tendency toward early discharge after uncomplicated deliveries in hospital [13]. This may increase the necessity of performing domiciliary CCHD screening in high- and middle-income countries and good quality smartphone pulse oximeters could be applied for these settings.
CCHD screening has less priority in low-income countries because treatment options are limited [27]. However, since CCHD screening is based on the detection of hypoxemia, infants with a positive screen may have another potentially serious and treatable condition, such as neonatal sepsis [1-5]. Simple procedures for identifying infants with infection that need referral for treatment are of major public health importance [28]. Providing a more extensive access to pulse oximetry in the first hours of life may reduce neonatal mortality and morbidity by early detection of hypoxemia in conditions such as sepsis, pneumonia, or other infections [2, 27].

As claimed by several CCHD-screening advocates, the question is not whether we should implement an inexpensive, quick, painless screen with the potential to save lives, but how it should be implemented $[4,29]$. Smartphones are powerful tools that offer both computational and communication opportunities which can be leveraged for the benefit of health care [17, 30]. Our study suggests that one of these benefits could be the extension of the screening of CCHD, as well as other forms of clinically undetectable hypoxemia, to a larger neonatal population.

\section{Disclosure Statement}

The authors declare no conflicts of interest.

\section{References}

1 Thangaratinam S, Brown K, Zamora J, Khan KS, Ewer AK: Pulse oximetry screening for critical congenital heart defects in asymptomatic newborn babies: a systematic review and meta-analysis. Lancet 2012;379:2459-2464.

2 Zhao Q-M, Ma X-J, Ge X-l, Liu F, Yan W-L, Wu L, Ye M, Liang X-C, Zhang J, Gao Y: Pulse oximetry with clinical assessment to screen for congenital heart disease in neonates in China: a prospective study. Lancet 2014;384: 747-754.

3 Ewer AK: Pulse oximetry screening: do we have enough evidence now? Lancet 2014;384: 725-726.

4 Ewer AK: Pulse oximetry screening for critical congenital heart defects in newborn infants: should it be routine? Arch Dis Child Fetal Neonatal Ed 2014;99:F93-F95.

5 Hoffman JI: It is time for routine neonatal screening by pulse oximetry. Neonatology 2010;99:1-9.
6 Narayen IC, Blom NA, Ewer AK, Vento M, Manzoni P, te Pas AB: 2015 Aspects of pulse oximetry screening for critical congenital heart defects: when, how and why? Arch Dis Child Fetal Neonatal Ed 2016;101:F162F167.

7 de-Wahl Granelli A, Meberg A, Ojala T, Steensberg J, Oskarsson G, Mellander M: Nordic pulse oximetry screening - implementation status and proposal for uniform guidelines. Acta Paediatr 2014;103:11361142.

8 Wong KK, Fournier A, Fruitman DS, Graves L, Human DG, Narvey M, Russell JL: Canadian Cardiovascular Society/Canadian Pediatric Cardiology Association position statement on pulse oximetry screening in newborns to enhance detection of critical congenital heart disease. Can J Cardiol 2016; 33:199-208.
9 Cloete E, Gentles T, Alsweiler J, Dixon L, Webster D, Rowe D, Bloomfield FH: Should New Zealand introduce nationwide pulse oximetry screening for the detection of critical congenital heart disease in newborn infants? N Z Med J 2017;130:64-69.

10 Martin GR, Beekman RH, Mikula EB, Fasules J, Garg LF, Kemper AR, Morrow WR, Pearson GD, Mahle WT: Implementing recommended screening for critical congenital heart disease. Pediatrics 2013;132:e185-e192.

11 Ewer AK, Granelli AD-W, Manzoni P, Luna MS, Martin GR: Pulse oximetry screening for congenital heart defects. Lancet 2013;382: 856-857.

12 Kemper AR, Mahle WT, Martin GR, Cooley WC, Kumar P, Morrow WR, Kelm K, Pearson GD, Glidewell J, Grosse SD: Strategies for implementing screening for critical congenital heart disease. Pediatrics 2011;128:e1259e1267. 
13 Narayen IC, Blom NA, Bourgonje MS, Haak MC, Smit M, Posthumus F, Van Den Broek AJ, Havers HM, te Pas AB: Pulse oximetry screening for critical congenital heart disease after home birth and early discharge. J Pediatr 2016;170:188-192.e1.

14 Narayen IC, Blom NA, Verhart MS, Smit M, Posthumus F, van den Broek AJ, Havers H, Haak MC, te Pas AB: Adapted protocol for pulse oximetry screening for congenital heart defects in a country with homebirths. Eur J Pediatr 2015;174:129-132.

15 Petersen CL, Gan H, Gorges M, Ansermino J, Dumont G: Systematic evaluation of mobile phone pulse oximetry performance. Proceedings of the Annual Meeting of the American Society of Anesthesiologists, San Francisco, 2013, vol 1216.

16 von Dadelszen P, Ansermino JM, Dumont G, Hofmeyr GJ, Magee LA, Mathai M, Sawchuck D, Teela K, Donnay F, Roberts JM: Improving maternal and perinatal outcomes in the hypertensive disorders of pregnancy: a vision of a community-focused approach. Int J Gynaecol Obstet 2012;119:S30-S34.

17 Atcheson CL: The future of anesthesiology and global health in a connected world; in Roth R, Frost EAM, Gevirtz C, Atcheson CL (eds): The Role of Anesthesiology in Global Health. Cham, Springer, 2015, pp 403-416.
18 Sola A: Oxygen saturation in the newborn and the importance of avoiding hyperoxia-induced damage. NeoReviews 2015;16:e393e405.

19 “iSpO2RX Pulse Oximeter". Retrieved 26 January 2017 from http://www.masimo.co.uk/ pulseOximeter/iSpO2Rx.htm.

20 Bland JM: How can I decide the sample size for a study of agreement between two methods of measurement? 2004. http://martinbland.co.uk/ (accessed January 26, 2017).

21 Bland JM, Altman D: Statistical methods for assessing agreement between two methods of clinical measurement. Lancet 1986;327:307310.

22 Lindholm C, Altimiras J: Point-of-care devices for physiological measurements in field conditions. A smorgasbord of instruments and validation procedures. Comp Biochem Physiol A 2016;202:99-111.

23 Cawsey M, Noble S, Cross-Sudworth F, Ewer A: Feasibility of pulse oximetry screening for critical congenital heart defects in homebirths. Arch Dis Child Fetal Neonatal Ed 2016;101:F349-F351.

24 Snowden JM, Tilden EL, Snyder J, Quigley B, Caughey $\mathrm{AB}$, Cheng YW: Planned out-ofhospital birth and birth outcomes. N Engl J Med 2015;373:2642-2653.
25 Pirneskoski J, Peräjoki K, Nuutila M, Kuisma M: Urgent EMS managed out-of-hospital delivery dispatches in Helsinki. Scand J Trauma Resusc Emerg Med 2016;24:94.

26 Blix E, Kumle MH, Ingversen K, Huitfeldt AS, Hegaard HK, Ólafsdóttir ÓÁ, Øian P, Lindgren $\mathrm{H}$ : Transfers to hospital in planned home birth in four Nordic countries - a prospective cohort study. Acta Obstet Gynecol Scand 2016;95:420-428.

27 King EM, Lieu C, Kasasa A, Ewer AK, Thangaratinam S: Pulse oximetry as a screening tool to detect hypoxia associated with earlyonset sepsis in asymptomatic newborns: a feasibility study in a low-income country. Br J Med Med Res 2014;4:1115-1128.

28 Weber MW, Carlin JB, Gatchalian S, Lehmann D, Muhe L, Mulholland EK; WHO Young Infants Study Group: Predictors of neonatal sepsis in developing countries. Pediatr Infect Dis J 2003;22:711-717.

29 Hom LA, Martin GR: US international efforts on critical congenital heart disease screening: can we have a uniform recommendation for Europe? Early Hum Dev 2014;90:S11-S14.

30 Behar J, Roebuck A, Domingos JS, Gederi E, Clifford GD: A review of current sleep screening applications for smartphones. Physiol Meas 2013;34:R29-R46. 